\title{
ON THE SPACE OF LIPSCHITZ HOMEOMORPHISMS OF A COMPACT POLYHEDRON
}

\author{
Katsuro Sakai and Raymond Y. Wong
}

\begin{abstract}
Let $X$ be a positive dimensional compact Euclidean polyhedron. Let $H(X), H_{\mathrm{LIP}}(X)$ and $H_{\mathrm{PL}}(X)$ be respectively the space of homeomorphisms, the space of Lipschitz homeomorphisms and the space of piecewise-linear homeomorphisms of $X$ onto itself. In this paper, we establish a homeomorphism taking the triple $\left(H(X), H_{\mathrm{LIP}}(X)\right.$, $\left.H_{\mathrm{PL}}(X)\right)$ onto the triple $\left(H(X) \times s, H_{\mathrm{LIP}}(X) \times \Sigma, H_{\mathrm{PL}}(X) \times \sigma\right)$, where $s=(-1,1)^{\omega}, \Sigma=\left\{\left(x_{i}\right) \in s|\sup | x_{i} \mid<1\right\}$ and $\sigma=\left\{\left(x_{i}\right) \in s \mid x_{i}=0\right.$ except for finitely many $i\}$. As a consequence we prove that when $X$ is a PL manifold with $\operatorname{dim} x \neq 4$ and $\partial X=\varnothing$, in case $\operatorname{dim} X=5$, $\left(H(X), H_{\mathrm{LIP}}(X)\right)$ is an $(s, \Sigma)$-manifold pair if $H(X)$ is an $s$-manifold. We also prove that if $\operatorname{dim} X=1$ or 2 , then $\left(H(X), H_{\mathrm{PL}}(X)\right)$ is an $(s, \sigma)$-manifold pair and $\left(H(X), H_{\mathrm{LIP}}(X)\right)$ is an $(s, \Sigma)$-manifold.
\end{abstract}

0. Introduction. Let $X=(X, d)$ and $Y=(Y, \rho)$ be metric spaces. A map $f: X \rightarrow Y$ is said to be Lipschitz (resp. bi-Lipschitz) if there is some $k \geq 0$ (resp. $k \geq 1$ ) such that $\rho\left(f(x), f\left(x^{\prime}\right)\right) \leq k \cdot d\left(x, x^{\prime}\right)$ (resp. $\left.k^{-1} \cdot d\left(x, x^{\prime}\right) \leq \rho\left(f(x), f\left(x^{\prime}\right)\right) \leq k \cdot d\left(x, x^{\prime}\right)\right)$ for all $x, x^{\prime} \in X$. The minimum of such $k \geq 0$ (resp. $k \geq 1$ ) is called the Lipschitz (resp. bi-Lipschitz) constant of $f$ and denoted by $\operatorname{lip}(f)$ (resp. bilip $(f)$ ). If $\operatorname{lip}(f) \leq k$ (resp. bilip $(f) \leq k), f$ is said to be $k$-Lipschitz (resp. $k$-biLipschitz). A bi-Lipschitz map is also called a Lipschitz embedding. A homeomorphism $f: X \rightarrow Y$ is called a Lipschitz homeomorphism if both $f$ and $f^{-1}$ are Lipschitz, namely, $f$ is bi-Lipschitz. Then $\operatorname{bilip}(f)=\max \left\{\operatorname{lip}(f), \operatorname{lip}\left(f^{-1}\right)\right\}$.

In this paper, we deal mainly with the cases where $X$ and $Y$ are positive dimensional Euclidean polyhedra and $X$ is compact. The space of all (continuous) maps from $X$ to $Y$ is denoted by $C(X, Y)$, with the topology of $C(X, Y)$ induced by the sup-metric $\rho(f, g)=$ $\sup \{\rho(f(x), g(x)) \mid x \in X\}$. By $\operatorname{LIP}(X, Y)$ and $\operatorname{PL}(X, Y)$, we denote the subspaces of $C(X, Y)$ consisting of all Lipschitz maps and PL maps, respectively. By $E(X, Y), E_{\mathrm{LIP}}(X, Y)$, and $E_{\mathrm{PL}}(X, Y)$, we denote the subspaces of $C(X, Y)$ consisting of all embeddings, all Lipschitz embeddings and all PL embeddings. Finally by $H(X), H_{\mathrm{LIP}}(X)$ and $H_{\mathrm{PL}}(X)$, we denote respectively the spaces of all homeomorphisms, all 
Lipschitz homeomorphisms and all PL homeomorphisms of $X$ onto itself (as subspaces of $C(X, X)$ ).

A paracompact (topological) manifold modeled on a given space $E$ is called an $E$-manifold. For $F \subset E$, an $(E, F)$-manifold pair is a pair $(M, N)$ of an $E$-manifold $M$ and an $F$-manifold $N$ which admits an open cover $\mathscr{U}$ of $M$ and open embeddings $\phi_{U}: U \rightarrow E, U \in \mathscr{U}$, such that $\phi_{U}(N \cap U)=F \cap \phi_{U}(U)$. For $G \subset F \subset E$, an $(E, F, C)$-manifold triple can be defined in similar manner. Let $Q$ denote the Hilbert cube $[-1,1]^{\omega}, s=(-1,1)^{\infty}, \Sigma=\left\{\left(x_{i}\right) \in S|\sup | x_{i} \mid<1\right\}$ and $\sigma=\left\{\left(x_{i}\right) \in\right.$ $s \mid x_{i}=0$ except for finitely many $\left.i\right\}$. By $\left[\mathbf{A n}_{1}\right] s$ is homeomorphic to $(\cong)$ the separable Hilbert space $l_{2}$. Denote $l_{2}^{Q}=\left\{\left(x_{i}\right) \in l_{2}|\sup | i x_{i} \mid<\infty\right\}$ and $l_{2}^{f}=\left\{\left(x_{i}\right) \in l_{2} \mid x_{i}=0\right.$ except for finitely many $\left.i\right\}$. It is well known that $(s, \Sigma) \cong\left(l_{2}, l_{2}^{Q}\right),(s, \sigma) \cong\left(l_{2}, l_{2}^{f}\right)$ and $(Q, Q \backslash s) \cong(Q, \Sigma)$ [An $\mathbf{n}_{2}$ ]. It is also well known that $(M, N)$ is an $(s, \Sigma)$-manifold (resp. $(s, \sigma)$-manifold) pair if and only if $M$ is an $s$-manifold and $N$ is a cap (resp. f.d. cap) set for $M$, and that $(M, N)$ is a $(Q, \Sigma)$-manifold (resp. $(Q, \sigma)$-manifold) pair if and only if $M$ is a $Q$-manifold and $N$ is a cap (resp. f.d. cap) set for $M$. For the definition of (f.d.) cap sets and related results, we refer to $[\mathbf{C h}]$. The same type of characterizations of $(s, \Sigma, \sigma)$-manifold triples and $(Q, \Sigma, \sigma)$-manifold triples are given in [SW] and, as an application, we show that $(s, \Sigma, \sigma) \cong\left(l_{2}, l_{2}^{Q}, l_{2}^{f}\right)$.

In $\S 1$, we prove a stability theorem for the triple $\left(H(X), H_{\mathrm{LIP}}(X)\right.$, $\left.H_{\mathrm{PL}}(X)\right)$; that is,

$$
\left(H(X) \times s, H_{\mathrm{LIP}}(X) \times \Sigma, H_{\mathrm{PL}}(X) \times \sigma\right) \cong\left(H(X), H_{\mathrm{LIP}}(X), H_{\mathrm{PL}}(X)\right),
$$

where $X$ is a compact polyhedron in $\mathbf{R}^{n}$ with positive dimension. We also establish a similar stability theorem for the triple $(E(X, Y)$, $\left.E_{\mathrm{LIP}}(X, Y), E_{\mathrm{PL}}(X, Y)\right)$.

In $\S 2$, it is shown that for a compact PL manifold $X$ in $\mathbf{R}^{n}$ with $\operatorname{dim} X \neq 4$ and $\partial X=\varnothing$ in case $\operatorname{dim} X=5,\left(H(X), H_{\mathrm{LIP}}(X)\right)$ is an $(s, \Sigma)$-manifold pair if $H(X)$ is an $s$-manifold (Theorem 2.3). Since it is known that when $\operatorname{dim} X=1$ or $2, H(X)$ is an $s$-manifold ([An $\mathbf{A n}_{3}$, [LM] and [To]), $\left(H(X), H_{\mathrm{LIP}}(X)\right)$ is an $(s, \Sigma)$-manifold pair. In fact, this is true for any compact polyhedron $X$ in $\mathbf{R}^{n}$ with $\operatorname{dim} X=1$ or 2 (Theorem 2.4).

Let $d$ and $d^{\prime}$ be metrics on $X$. We say that $d$ and $d^{\prime}$ are Lipschitz equivalent if the identity map id: $(X, d) \rightarrow\left(X, d^{\prime}\right)$ is a Lipschitz homeomorphism.

The authors would like to thank Jouni Luukkainen for carefully reading the manuscript. 
1. The $\Sigma$-stability of $H_{\mathrm{LIP}}(X)$ and $E_{\mathrm{LIP}}(X, Y)$. In this section, we prove the following stability theorems:

1.1. Theorem. For any positive dimensional compact Euclidean polyhedron $X$,

$$
\left(H(X) \times s, H_{\mathrm{LIP}}(X) \times \Sigma, H_{\mathrm{PL}}(X) \times \sigma\right) \cong\left(H(X), H_{\mathrm{LIP}}(X), H_{\mathrm{PL}}(X)\right) .
$$

1.2. Theorem. Let $X$ and $Y$ be positive dimensional Euclidean polyhedra where $X$ is compact. If $E_{\mathrm{PL}}(X, Y) \neq \varnothing$ then

$$
\begin{gathered}
\left(E(X, Y) \times s, E_{\mathrm{LIP}}(X, Y) \times \Sigma, E_{\mathrm{PL}}(X, Y) \times \sigma\right) \\
\cong\left(E(X, Y), E_{\mathrm{LIP}}(X, Y), E_{\mathrm{PL}}(X, Y)\right) .
\end{gathered}
$$

To prove Theorem 1.1, we first establish several lemmas. The procedure employed here is a modification of some technique developed by [K-W] in studying $H_{\mathrm{PL}}(X)$. These are put together in a non-trivial way to obtain the result stated in Theorem 1.1. (The proof of Theorem 1.2 is entirely analogous to Theorem 1.1 and therefore will be omitted.)

First, let us recall the definition of Morse's $\mu$-length of paths [Mo] (cf. [ $\left.\left.\mathbf{G e}_{1}\right]\right)$. Let $f:[a, b] \rightarrow X$ be a path where $a<b \in \mathbf{R}$. For each $n \in \mathbf{N}$, let $A_{n}=\left\{\left(t_{0}, t_{1}, \ldots, t_{n}\right) \mid a \leq t_{0} \leq \cdots \leq t_{n} \leq b\right\}$. Define

$$
\delta\left(f ; t_{0}, \ldots, t_{n}\right)=\min \left\{d\left(f\left(t_{i}\right), f\left(t_{i-1}\right)\right) \mid i=1, \ldots, n\right\}
$$

for each $\left(t_{0}, \ldots, t_{n}\right) \in A_{n}$ and

$$
\mu_{n}(f)=\sup \left\{\delta\left(f ; t_{0}, \ldots, t_{n}\right) \mid\left(t_{0}, \ldots, t_{n}\right) \in A_{n}\right\} .
$$

The $\mu$-length of $f$ is defined as $\mu(f)=\sum_{n=1}^{\infty} 2^{-n} \mu_{n}(f)$. Then $\mu$ : $C([a, b], X) \rightarrow[0, \infty)$ is continuous. The following is obvious from the definition.

(0) Given $f \in E([a, b], X), f^{\prime} \in E\left(\left[a^{\prime}, b^{\prime}\right], X\right)$, if $f([a, b]) \subset$ $f^{\prime}\left(\left[a^{\prime}, b^{\prime}\right]\right)$ then $\mu(f) \leq \mu\left(f^{\prime}\right)$.

1.3. LemMA. There exists a map $\tau: E([-1,1], X) \rightarrow(-1,1)$ having the following properties:

(1) $\tau(f)=t$ if and only if $\mu(f \mid[-1, t])=\mu(f \mid[t, 1])$,

(2) $\tau(f)=0$ if $f$ is linear (affine).

Proof. Define $\tilde{\gamma}: E([-1,1], X) \times[-1,1] \rightarrow[-1,1]$ by

$$
\tilde{\gamma}(f, t)=\mu(f)^{-1}(\mu(f \mid[-1, t])-\mu(f \mid[t, 1])) .
$$


By similar argument as in [ $\mathbf{G e}_{1}$, Corollary 1.6], we can show that $\widetilde{\gamma}$ is continuous. For each $f \in([-1,1], X)$, let $\gamma(f):[-1,1] \rightarrow[-1,1]$ be the map defined by $\gamma(f)(t)=\tilde{\gamma}(f, t)$. Then $\gamma(f) \in H([-1,1])$ since $\gamma(f)(t)<\gamma(f)\left(t^{\prime}\right)$ for $t<t^{\prime}$ and $\gamma(f)( \pm 1)= \pm 1$. Thus we have a map $\gamma: E([-1,1], X) \rightarrow H([-1,1])$. Finally we define $\tau: E([-1,1], X) \rightarrow$ $(-1,1)$ by $\tau(f)=\gamma(f)^{-1}(0)$. It is obvious $\tau$ satisfies properties (1) and (2) as required.

The following lemma is a consequence of [ $\mathbf{L V}$, Lemma 2.22].

1.4. Lemma. Let $f: C \rightarrow Y$ be a function from a convex set $C$ in a normed linear space to a metric space $Y, A$ a closed convex subset of $C$ and $B=C \backslash \operatorname{int}_{C} A$. If $f \mid A$ and $f \mid B$ are Lipschitz then so is $f$ and $\operatorname{lip}(f)=\max \{\operatorname{lip}(f \mid A), \operatorname{lip}(f \mid B)\}$.

Let us consider the norm $\|x\|_{1}=\left|x_{1}\right|+\cdots+\left|x_{n}\right|$ on $\mathbf{R}^{n}$, which is Lipschitz equivalent to the Euclidean norm $\|x\|=\left(x_{1}^{2}+\cdots+x_{n}^{2}\right)^{1 / 2}$. Let $B_{1}^{n}=\left\{x \in \mathbf{R}^{n} \mid\|x\|_{1} \leq 1\right\}$. Then $B_{1}^{n}$ is a convex polyhedron. We should remark $H_{\mathrm{PL}}\left(B_{1}^{n}\right) \subset H_{\mathrm{LIP}}\left(B_{1}^{n},\|\cdot\|\right)=H_{\mathrm{LIP}}\left(B_{1}^{n},\|\cdot\|_{1}\right)$. Let $e_{n}=(0, \ldots, 0,1) \in \mathbf{R}^{n}$ and $\alpha:[-1,1] \rightarrow B_{1}^{n}$ be the arc defined by $\alpha(t)=t \cdot e_{n}$. For each $i \in \mathbf{N}$, let $\alpha_{i}:[-1,1] \rightarrow B_{1}^{n}$ be the arc defined by $\alpha_{i}(t)=\alpha\left(2^{-(i+2)}(t+4)\right)$.

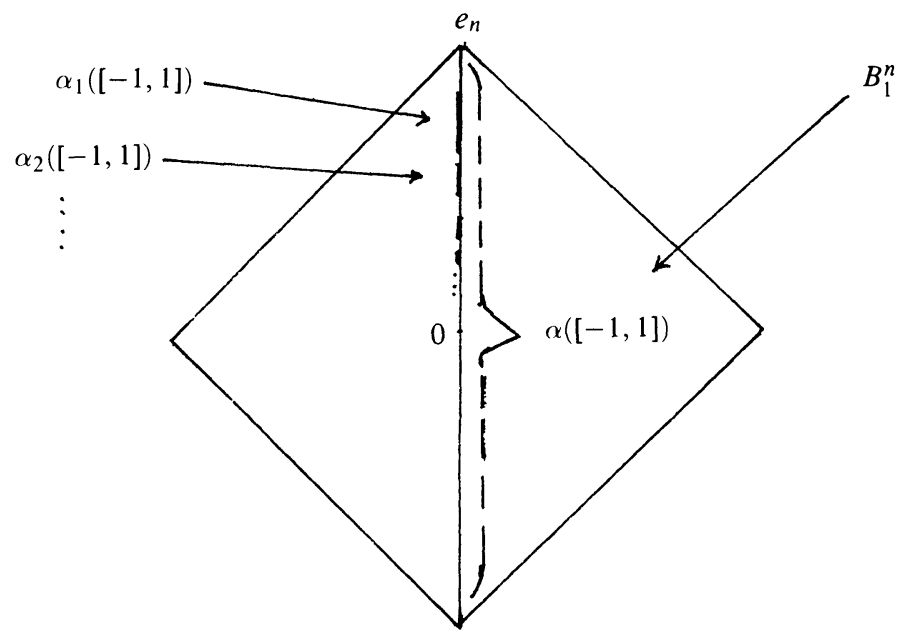

Figure 1

1.5. Lemma. There exists a map

$$
\xi:(s, \Sigma, \sigma) \rightarrow\left(H\left(B_{1}^{n}\right), H_{\mathrm{LIP}}\left(B_{1}^{n}\right), H_{\mathrm{PL}}\left(B_{1}^{n}\right)\right)
$$


such that for each $z \in s$ and $i \in \mathbf{N}$,

(3) $\xi(z) \mid B_{1}^{n} \backslash \frac{3}{4} B_{1}^{n}=\mathrm{id}$,

(4) $\xi(z)\left(\alpha_{i}([-1,0])\right)=\alpha_{i}\left(\left[-1, z_{i}\right]\right)$ and

(5) $\xi(z)\left(\alpha_{i}([0,1])\right)=\alpha_{i}\left(\left[z_{i}, 1\right]\right)$.

Proof. For each $t \in(-1,1)$, let $\theta(t) \in H_{\mathrm{PL}}\left(B_{1}^{n}\right)$ be such that $\theta(t)$ maps the straight line segment between $b \in \partial B_{1}^{n}$ and 0 linearly onto the one between $b$ and $t e_{n}$, that is,

$$
\begin{aligned}
\theta(t)(x) & =x+t\left(1-\|x\|_{1}\right) \cdot e_{n} \\
& =\left(x_{1}, \ldots, x_{n-1}, t\left(1-\left|x_{1}\right|-\cdots-\left|x_{n}\right|\right)\right) .
\end{aligned}
$$

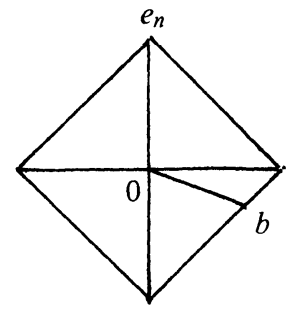

$B_{1}^{n}$

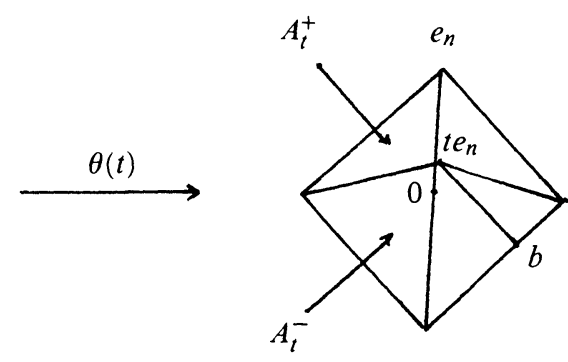

$B_{1}^{n}$

FiguRe 2

Then clearly $\theta:(-1,1) \rightarrow H_{\mathrm{PL}}\left(B_{1}^{n}\right)$ is continuous and $\theta(0)=\mathrm{id}$. For each $y \in B_{1}^{n}$ and $t \in(-1,1)$. Let $\zeta(y, t)=y_{n}-t\left(1-\left|y_{1}\right|-\cdots-\left|y_{n-1}\right|\right)$. Let $A_{t}^{-}\left\{y \in B_{1}^{n} \mid \zeta(y, t) \geq 0\right\}$ and $A_{t}^{-}=\left\{y \in B_{1}^{n} \mid \zeta(y, t) \leq 0\right\}$. We observe that

$$
\theta(t)^{-1}(y)= \begin{cases}\left(y_{1}, \ldots, y_{n-1},(1-t)^{-1} \zeta(y, t)\right) & \text { if } y \in A_{t}^{+} \\ \left(y_{1}, \ldots, y_{n-1},(t+1)^{-1} \zeta(y, t)\right) & \text { if } y \in A_{t}^{-}\end{cases}
$$

We first want to show $\operatorname{bilip}(\theta(t))=(1-|t|)^{-1}$ : For each $x, x^{\prime} \in B_{1}^{n}$,

$$
\begin{aligned}
\left\|\theta(t)(x)-\theta\left(x^{\prime}\right)\right\|_{1} & \leq\left\|x-x^{\prime}\right\|_{1}+|t| \cdot\left|,\|x\|_{1}-\left\|x^{\prime}\right\|_{1}\right| \\
& \leq(1-|t|)\left\|x-x^{\prime}\right\|_{1},
\end{aligned}
$$




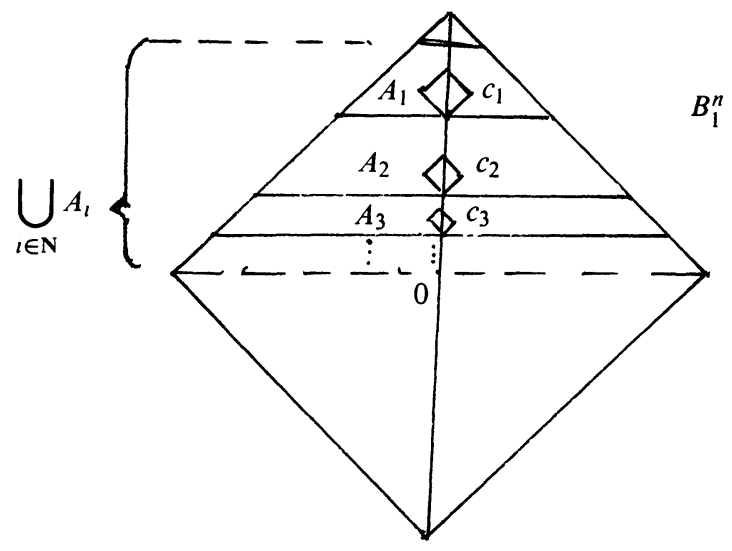

Figure 3

which implies $\operatorname{lip}(\theta(t)) \leq 1+|t|$. For each $y, y^{\prime} \in B_{1}^{n}$, if $\zeta(y, t)$. $\zeta\left(y^{\prime}, t^{\prime}\right) \geq 0$ then

$$
\begin{aligned}
&\left\|\theta(t)^{-1}(y)-\theta(t)^{-1}\left(y^{\prime}\right)\right\|_{1} \\
& \leq\left|y_{1}-y_{1}^{\prime}\right|+\cdots+\left|y_{n-1}-y_{n-1}^{\prime}\right| \\
&+(1-|t|)^{-1}\left(\left|y_{n}-y_{n}^{\prime}\right|+|t|\left(|| y_{1}|-| y_{1}^{\prime}||+\cdots+|| y_{n-1}|-| y_{n-1}^{\prime}||\right)\right) \\
& \leq(1-|t|)^{-1}\left(\left|y_{1}-y_{1}^{\prime}\right|+\cdots+\left|y_{n}-y_{n}^{\prime}\right|\right) \\
&=(1-|t|)^{-1}|| y-y^{\prime} \|_{1} .
\end{aligned}
$$

We observe that $A_{t}^{+}$is convex if $t \leq 0$ and $A_{t}^{-}$is convex if $t \geq 0$. Then by Lemma 1.4, $\operatorname{lip}\left(\theta(t)^{-1}\right) \leq(1-|t|)^{-1}$. We have

$$
\left\|\theta(t)(0)-\theta(t)\left(e_{n}\right)\right\|_{1}=1-|t| \quad \text { if } t \geq 0
$$

and

$$
\left\|\theta(t)(0)-\theta(t)\left(-e_{n}\right)\right\|_{1}=1-|t| \quad \text { if } t<0 .
$$

Therefore $\operatorname{lip}\left(\theta(t)^{-1}\right)=(1-|t|)^{-1}$. Since $1+|t| \leq(1-|t|)^{-1}$,

$$
\operatorname{bilip}(\theta(t))=\max \left\{\operatorname{lip}(\theta(t)), \operatorname{lip}(\theta(t))^{-1}\right\}=(1-|t|)^{-1} .
$$

For each $i \in \mathbf{N}$, let $\phi_{i}: \mathbf{R}^{n} \rightarrow \mathbf{R}^{n}$ be the linear (affine) homeomorphism defined by $\phi_{i}(x)=w^{-(i+2)}\left(x+4 e_{n}\right)$ and let $C_{i}=\phi_{i}\left(B_{1}^{n}\right)$. Then $\alpha_{i}=\phi_{i} \cdot \alpha$ for each $i \in \mathbf{N}$. For each $z \in S$, we define $\xi(z) \in H\left(B_{1}^{n}\right)$ as follows:

$$
\begin{gathered}
\xi(z) \mid C_{i}=\phi_{i} \cdot \theta\left(z_{1}\right) \cdot \phi_{i}^{-1} \in H_{\mathrm{PL}}\left(C_{i}\right) \quad \text { for } i \in \mathbf{N}, \\
\xi(z) \mid B_{1}^{n} \backslash \bigcup_{i \in \mathbf{N}} C_{i}=\mathrm{id} .
\end{gathered}
$$

For each $z \in \sigma, \xi(z) \mid C_{i}=$ id except for finitely many $i \in \mathbf{N}$, which implies $\xi(z) \in H_{\mathrm{PL}}\left(B_{1}^{n}\right)$. Hence $\xi(\sigma) \subset H_{\mathrm{PL}}\left(B_{1}^{n}\right)$. 
Finally we will show $\xi(\Sigma) \subset H_{\mathrm{LIP}}\left(B_{1}^{n}\right)$ :

For each $i \in \mathbf{N}$, let

$$
A_{i}=\left\{x \in B_{1}^{n} \mid 2^{-(i+2)} \cdot 3 \leq x_{n} \leq 2^{-(i+1)} \cdot 3\right\} .
$$

It follows from Lemma 1.4 that for each $z \in \Sigma$ and $i \in \mathbf{N}, \xi(z) \mid A_{i} \in$ $H_{\mathrm{LIP}}\left(A_{i}\right)$ and

$$
\begin{aligned}
\operatorname{bilip}\left(\xi(z) \mid A_{i}\right) & =\operatorname{bilip}\left(\xi(z) \mid C_{i}\right)=\operatorname{bilip}\left(\phi_{i} \cdot \theta\left(z_{i}\right) \cdot \phi_{i}^{-1}\right) \\
& =\operatorname{bilip}\left(\theta\left(z_{i}\right)\right)=\left(1-\left|z_{1}\right|\right)^{-1} \leq\left(1-\sup _{j \in \mathbf{N}}\left|z_{j}\right|\right)^{-1} .
\end{aligned}
$$

By using Lemma 1.4 inductively, we can verify

$$
\xi(z) \mid \bigcup_{i \in \mathbf{N}} A_{i} \in H_{\mathrm{LIP}}\left(\bigcup_{i \in \mathbf{N}} A_{i}\right)
$$

and

$$
\operatorname{bilip}\left(\xi(z) \mid \bigcup_{i \in \mathbf{N}} A_{i}\right)=\left(1-\sup \left|z_{i}\right|\right)^{-1}
$$

It is clear that if $f$ i s $k$-Lipschitz when restricted to a dense subset of $X, f$ is itself $k$-Lipschitz. Using this and Lemma 1.4, we have $\xi(z) \in H_{\mathrm{LIP}}\left(B_{1}^{n}\right)$ with $\operatorname{bilip}(\xi(z))=\left(1-\sup \left|z_{i}\right|\right)^{-1}$. Thus we have a map

$$
\xi:(s, \Sigma, \sigma) \rightarrow\left(H\left(B_{1}^{n}\right), H_{\mathrm{LIP}}\left(B_{1}^{n}\right), H_{\mathrm{PL}}\left(B_{1}^{n}\right)\right) .
$$

From the definition, it is clear that $\xi$ satisfies the desired conditions.

Proof of Theorem 1.1. Let $C$ be a simplex in $X$ with $\operatorname{dim} C=$ $\operatorname{dim} X=n$ and $\beta: B_{1}^{n} \rightarrow C$ a linear embedding. We define a map $F: s \rightarrow H(X)$ by $F(z) \mid \beta\left(B_{1}^{n}\right)=\beta \cdot \xi(z) \cdot \beta^{-1}$ and $F(z) \mid X \backslash \beta\left(B_{1}^{n}\right)=$ id. Then for each $z \in \Sigma, F(z)$ and $F(z)^{-1}$ are (locally) Lipschitz by the definition and property (3) in Lemma 1.5. Hence $F(\Sigma) \subset H_{\mathrm{LIP}}(X)$ (clearly $\left.F(\sigma) \subset H_{\mathrm{PL}}(X)\right)$. Let $g=\beta \alpha, g_{i}=\beta \alpha_{i} \in E_{\mathrm{PL}}([-1,1], X)$, $i \in \mathbf{N}$. Then $g_{i}(t)=g\left(2^{-(i+2)}(t+4)\right)$. From (4) and (5), we have

(6) $F(z) \cdot g_{i}([-1,0])=g_{i}\left(\left[-1, z_{i}\right]\right)$ and

(7) $F(z) \cdot g_{i}([0,1])=g_{i}\left(\left[z_{i}, 1\right]\right)$

for each $z \in s$ and $i \in \mathbf{N}$.

We define a map $T: H(x) \rightarrow s$ by $T(h)=\left(\tau\left(h g_{i}\right)\right)_{i \in \mathbf{N}}$. For each $h \in H_{\mathrm{PL}}(X), h g_{i}$ is linear except for finitely many $i \in \mathbf{N}$, which implies $T(h) \in \sigma$ by (2). Hence $T\left(H_{\mathrm{PL}}(X)\right) \subset \sigma$. We will show that $T\left(H_{\mathrm{LIP}}(X)\right) \subset \Sigma$. To this end, let $h \in H_{\mathrm{LIP}}(X)$ and $T(h)=z$. Then for 
each $i \in \mathbf{N}, \tau\left(h g_{i}\right)=z_{i}$, which means $\mu\left(h g_{i} \mid\left[-1, z_{i}\right]\right)=\mu\left(h g_{i} \mid\left[z_{i}, 1\right]\right)$ by (1). Since $g_{i}(t)=g\left(2^{-(i+2)}(t+4)\right)$, we have by $(0)$

$$
\mu\left(h g \mid\left[2^{-(i+2)} \cdot 3,2^{-(i+2)}\left(z_{i}+r\right)\right]\right)=\mu\left(h g \mid\left[2^{-(i+2)}\left(z_{i}+4\right), 2^{-(i+2)} \cdot 5\right]\right) .
$$

Note that $h g \in E_{\mathrm{LIP}}([-1,1], X)$. Let $k=\operatorname{bilip}(h g) \geq 1$. From the definition of $\mu$-length, it is easy to verify that

$$
\begin{aligned}
\sum_{j=1}^{\infty} 2^{-j} \cdot k^{-1} \cdot 2^{-(i+2)} \cdot \frac{1+z_{i}}{j} \\
\quad \leq \mu\left(h g \mid\left[2^{-(i+2)} \cdot 3,2^{-(i+2)}\left(z_{i}+4\right)\right]\right) \\
\quad \leq \sum_{j=1}^{\infty} 2^{-j} \cdot k \cdot 2^{-(i+2)} \cdot \frac{1+z_{i}}{j}
\end{aligned}
$$

and

$$
\begin{aligned}
\sum_{j=1}^{\infty} 2^{-j} \cdot k^{-1} \cdot 2^{-(i+2)} \cdot \frac{1-z_{i}}{j} \\
\quad \leq \mu\left(h g \mid\left[2^{-(i+2)}\left(z_{i}+4\right), 2^{-(i+2)} \cdot 5\right]\right) \\
\quad \leq \sum_{j=1}^{\infty} 2^{-j} \cdot k \cdot 2^{-(i+2)} \cdot \frac{1-z_{i}}{j} .
\end{aligned}
$$

It follows that $l^{-1}\left(1+z_{i}\right) \leq k\left(1-z_{i}\right)$ and $k^{-1}\left(1-z_{1}\right) \leq k\left(1+z_{i}\right)$. This yields $\left|z_{i}\right| \leq\left(k_{1}^{2}\right) /\left(k^{2}+1\right)<1$. Therefore $T(h)=z \in \Sigma$.

Let $H^{0}(X)=T^{-1}(0), H_{\mathrm{LIP}}^{0}(X)=H_{\mathrm{LIP}}(X) \cap T^{-1}(0)$ and $H_{\mathrm{PL}}^{0}(X)=$ $H_{\mathrm{PL}}(X) \cap T^{-1}(0)$. Then $h \cdot F T(h) \in H^{0}(X)$ for each $h \in H(X)$. In fact, let $T(h)=z$; that is, $\tau\left(h g_{i}\right)=z_{i}$ for each $i \in \mathbf{N}$. Then $\mu\left(h g_{i} \mid\left[-1, z_{i}\right]\right)=\mu\left(g h_{i} \mid\left[z_{i}, 1\right]\right)$ by (1). From (6) and (7),

$$
\begin{aligned}
& h \cdot F T(h) \cdot g_{i}([-1,0])=h g_{i}\left(\left[-1, z_{i}\right]\right) \text { and } \\
& h \cdot F T(h) \cdot g_{i}([0,1])=h g_{i}\left(\left[z_{i}, 1\right]\right) .
\end{aligned}
$$

By (0), we have

$$
\begin{aligned}
& \mu\left(h \cdot F T(h) \cdot g_{i} \mid[-1,0]\right)=\mu\left(h g_{i} \mid\left[-1, z_{i}\right]\right) \\
& \quad=\mu\left(h g_{i} \mid\left[z_{i}, 1\right]\right)=\mu\left(h \cdot F T(h) \cdot g_{i} \mid[0,1]\right) .
\end{aligned}
$$

This means $\tau\left(h \cdot F T(h) \cdot g_{i}\right)=0$ by (1). Hence $T(h \cdot F T(h))=0$, namely $h \cdot F T(h) \in H^{0}(X)$. Thus we have a map $G: H(X) \rightarrow H^{0}(X)$ defined by $G(h)=h \cdot F T(h)$. Then $G\left(H_{\mathrm{LIP}}(X)\right) \subset H_{\mathrm{LIP}}^{0}(X)$ and $G\left(H_{\mathrm{PL}}(X)\right) \subset$ $H_{\mathrm{PL}}^{0}(X)$ since $F T\left(H_{\mathrm{LIP}}(X)\right) \subset F(\Sigma) \subset H_{\mathrm{LIP}}(X)$ and $F T\left(H_{\mathrm{PL}}(X)\right) \subset$ $F(\sigma) \subset H_{\mathrm{PL}}(X)$. 
Now we define maps

$$
\begin{aligned}
P:(H(X), & \left.H_{\mathrm{LIP}}(X), H_{\mathrm{PL}}(X)\right) \\
& \rightarrow\left(H^{0}(X) \times s, H_{\mathrm{LIP}}^{0}(X) \times \Sigma, H_{\mathrm{PL}}^{0}(X) \times \sigma\right), \\
Q:\left(H^{0}(X)\right. & \left.\times s, H_{\mathrm{LIP}}^{0}(X) \times \Sigma, H_{\mathrm{PL}}^{0}(X) \times \sigma\right) \\
\rightarrow & \left(H(X), H_{\mathrm{LIP}}(X), H_{\mathrm{PL}}(X)\right)
\end{aligned}
$$

by $P(h)=(G(h), T(h))$ and $Q(h, z)=h \cdot F(z)^{-1}$, where $h \in H(X)$. We will show that $P Q=$ id and $Q P=$ id. Let $h \in H^{0}(X)$ and $z \in s$. Then for each $i \in \mathbf{N}, \tau\left(h g_{i}\right)=0$, which means $\mu\left(h g_{i} \mid[-1,0]\right)=\mu\left(h g_{i} \mid[0,1]\right)$ by (1). From (6) and (7),

$$
\begin{aligned}
& h \cdot F(z)^{-1} \cdot g_{i}\left(\left[-1, z_{i}\right]\right)=h g_{i}([-1,0]) \quad \text { and } \\
& h \cdot F(z)^{-1} \cdot g_{i}\left(\left[z_{i}, 1\right]\right)=h g_{i}([0,1]) .
\end{aligned}
$$

By (0), we have

$$
\begin{aligned}
& \mu\left(h \cdot F(z)^{-1} \cdot g_{i} \mid\left[-1, z_{i}\right]\right)=\mu\left(h g_{i} \mid[-1,0]\right) \\
& \quad=\mu\left(h g_{i} \mid[0,1]\right)=\mu\left(h \cdot F(z)^{-1} \cdot g_{i} \mid\left[z_{i}, 1\right]\right) .
\end{aligned}
$$

This implies $\tau\left(h \cdot F(z)^{-1} \cdot g_{i}\right)=z_{i}$ by (1). Hence $T\left(h \cdot F(z)^{-1}\right)=z$. It follows that

$$
\begin{aligned}
G\left(h \cdot F(z)^{-1}\right) & =h \cdot F(z)^{-1} \cdot F T\left(h \cdot F(z)^{-1}\right) \\
& =h \cdot F(z)^{-1} \cdot F(z)=h .
\end{aligned}
$$

Therefore $P Q=$ id. On the other hand, let $h \in H(X)$. Then

$$
\begin{aligned}
Q P(h) & =Q(G(h), T(h))=G(h) \cdot F T(h)^{-1} \\
& =h \cdot F T(h) \cdot F T(h)^{-1}=h .
\end{aligned}
$$

Hence we have $Q P=$ id. Consequently

$$
\left(H^{0}(X) \times s, H_{\mathrm{LIP}}^{0}(X) \times \Sigma, H_{\mathrm{PL}}^{0}(X) \times \sigma\right) \cong\left(H(X), H_{\mathrm{LIP}}(X), H_{\mathrm{PL}}(X)\right) .
$$

Since $(s \times s, \Sigma \times \Sigma, \sigma \times \sigma) \cong(s, \Sigma, \sigma)$, we have the desired result.

A proof for Theorem 1.2 can be given entirely analogous to the proof of Theorem 1.1 and will be omitted.

Let $X$ be a compactum. Suppose there is a Lipschitz embedding $\beta: B_{1}^{n} \rightarrow X$ for some $n \geq 1$ such that $\beta\left(\stackrel{\circ}{B}_{1}^{n}\right)$ is open, where $\stackrel{\circ}{B}_{1}^{n}=\{x \in$ $\left.\mathbf{R}^{n} \mid\|x\|_{1}<1\right\}$; then the arguments in the proof of Theorem 1.1 can be applied to show

$$
\left(H(X) \times s, H_{\mathrm{LIP}}(X) \times \Sigma\right) \cong\left(H(X), H_{\mathrm{LIP}}(X)\right) .
$$


Hence we have the following stability theorem:

1.6. THEOREM. Let $X$ be a metric compactum. If $X$ has an open set which is Lipschitz homeomorphic to an open set in $\mathbf{R}^{n}$ for some $n \geq 1$, then

$$
\left(H(X) \times s, H_{\mathrm{LIP}}(X) \times \Sigma\right) \cong\left(H(X), H_{\mathrm{LIP}}(X)\right) .
$$

Similarly we have

1.7. Theorem. Let $X$ be a metric compactum and $Y$ a metric space. If $X$ has an open set which is Lipschitz homeomorphic to an open set in $\mathbf{R}^{n}$ for some $n \geq 1$ and $E_{\mathrm{LIP}}(X, Y) \neq \varnothing$, then

$$
\left(E(X, y) \times s, E_{\mathrm{LIP}}(X, Y) \times \Sigma\right) \cong\left(E(X, Y), E_{\mathrm{LIP}}(X, Y)\right) .
$$

2. The compact absorption property of $H_{\mathrm{LIP}}(X)$ for $H(X)$. First we remark that the Deformation Theorem in [SS] implies the following.

2.2. TheOREM. Let $X$ be a compact Euclidean polyhedron. Then $H_{\mathrm{LIP}}(X)$ is locally contractible.

2.2. Theorem. Let $X$ be a compact $\mathrm{PL}$ manifold in $\mathbf{R}^{m}$ with $\operatorname{dim} X$ $\neq 4$ and $\partial X=\varnothing$ in case $\operatorname{dim} X=5$. Then the closure of $H_{\mathrm{PL}}(X)$ in $H_{\mathrm{LIP}}(X)$ is the union of components of $H_{\mathrm{LIP}}(X)$.

Proof. As it was shown in the proof of [GH, Theorem 1], for each component $H$ of $H(X), H \cap H_{\mathrm{PL}}(X)$ is either dense in $H$ or empty. This yields the same property for $H_{\mathrm{PL}}(X) \subset H_{\mathrm{LIP}}(X)$. By uniform local contractibility of $H_{\mathrm{LIP}}(X)$, the same arguments as in the proof of [GH, Theorem 1] also show that the closure of $H_{\mathrm{PL}}(X)$ in $H_{\mathrm{LIP}}(X)$ is the union of components of $H_{\mathrm{LIP}}(X)$.

Let $X$ be a compact PL manifold in $\mathbf{R}^{m}$. Let $H^{*}(X)$ denote the subset of $H(X)$ consisting of those homeomorphisms which are isotopic to PL homeomorphisms and let $H_{\mathrm{LIP}}^{*}(X)=H^{*}(X) \cap H_{\mathrm{LIP}}(X)$.

2.3. Theorem. Let $X$ be compact PL manifold in $\mathbf{R}^{m}$ with $\operatorname{dim} X$ $\neq 4$, and $\partial X=\varnothing$ in case $\operatorname{dim} X=5$. Then $\left(H(X), H_{\mathrm{LIP}}(X)\right)$ is an $(s, \Sigma)$-manifold pair if $H(X)$ is an s-manifold .

Proof. Since $H_{\mathrm{PL}}(X)$ is a $\sigma$-compact ANR ([Ge 2$]$ and [Ha]), $H_{\mathrm{PL}}(X)$ $\times \Sigma$ is a $\Sigma$-manifold by the result of Torunczyk [To]. Then by Theorem 1.1 , we have a $\Sigma$-manifold $M$ with $H_{\mathrm{PL}}(X) \subset M \subset H_{\mathrm{LIP}}^{*}(X)$. By [GH, 
Theorem 2], $H_{\mathrm{PL}}(X)$ is an f.d. cap set for $H^{*}(X)$. Then for each $\varepsilon>0$ and each compact set $A \subset H^{*}(X)$, we have a map $h: A \rightarrow$ $M$ which is $\varepsilon$-close to id. By using a $\Sigma$-manifold version of [ $\left[\mathbf{S a}_{1}\right.$, Lemma 2], we can show that $M$ is a cap set for $H^{*}(X)$ (cf. [Sa 2 , Proof of Proposition 2.1]. If $H(X)$ is an $s$-manifold, so is $H^{*}(X)$ ([GH, Theorem 1]). By using Arzela-Ascoli's Theorem, it is easy to show that $H_{\mathrm{LIP}}(X)$ is $\sigma$-compact. Then $H_{\mathrm{LIP}}^{*}(X)$ is $\sigma$-compact by Theorem 1.2 , hence a $Z_{\sigma}$-set in $H^{*}(X)$. By [Ch, Theorem 6.6f], $H_{\mathrm{LIP}}^{*}(X)$ is a cap set for $H^{*}(X)$. Hence $\left(H^{*}(X), H_{\mathrm{LIP}}^{*}(X)\right)$ is an $(s, \Sigma)$-manifold pair. Since $H_{\mathrm{LIP}}(X)$ is dense in $H(X)$ [Su, Corollary 3] and $H(X)$ is homogeneous, $\left(H(X), H_{\mathrm{LIP}}(X)\right)$ is also an $(s, \Sigma)$-manifold pair.

It is well known that $H(X)$ is an $s$-manifold in case $\operatorname{dim} X=1$ or 2 ([An $\left.\mathbf{n}_{3}\right]$, [To]). Hence $\left(H(X), H_{\mathrm{LIP}}(X)\right)$ is an $(s, \Sigma)$-manifold pair. But in these dimensions it was also shown that $H(X)$ is an $s$-manifold

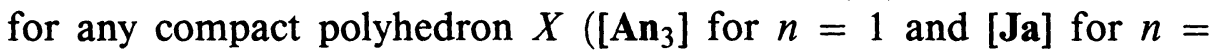
2). Furthermore, $H_{\mathrm{PL}}(X)$ is a $\sigma$-manifold ([Ja]) and dense in $H(X)$ ([Br]). Using uniform local contractibility of $H_{\mathrm{PL}}(X)$, the proof of [GH, Theorem 2] implies that $H_{\mathrm{PL}}(X)$ is an f.d. cap set for $H(X)$, that is, $\left(H(X), H_{\mathrm{PL}}(X)\right)$ is an $(s, \sigma)$-manifold pair. Applying the same arguments as in the proof of Theorem 2.3, $\left(H(X), H_{\mathrm{LIP}}(X)\right)$ is an $(s, \Sigma)$-manifold. We summarize the above remarks as

2.4. TheOREM. Let $X$ be a compact Euclidean polyhedron with $\operatorname{dim} X=1$ or 2 . Then $\left(H(X), H_{\mathrm{PL}}(X)\right)$ is an $(s, \sigma)$-manifold pair and $\left(H(X), H_{\mathrm{LIP}}(X)\right)$ is an $(s, \Sigma)$-manifold pair.

Actually, $H_{\mathrm{PL}}(X)$ can be shown to be a $\sigma$-manifold for any positive dimensional compact polyhedron $X$. We outline a proof of this fact as follows: $H_{\mathrm{PL}}(X)$ is locally contractible by [Ga]; hence $H_{\mathrm{PL}}(X)$ is a $\sigma-$ fd-compact ANR by [Ge $\mathbf{G e}_{2}$ and [Ha]. By [KW], $H_{\mathrm{PL}}(X) \times \sigma \cong H_{\mathrm{PL}}(X)$ (cf. Theorem 1.1). Hence $H_{\mathrm{PL}}(X)$ is a $\sigma$-manifold by [To].

Moreover it can be shown that $H_{\mathrm{PL}}(X)$ is an f.d. cap set for the closure $\overline{H_{\mathrm{PL}}(X)}$ of $H_{\mathrm{PL}}(X)$ in $H(X)$. In fact, for each finite-dimensional compact set $A \subset \overline{H_{\mathrm{PL}}(X)}$ and $\varepsilon>0$, it suffices to construct a map $f: A \rightarrow H_{\mathrm{PL}}(X)$ which is $\varepsilon$-close to id (cf. [Sa 2 , Proposition 2.1]). Using uniform local contractibility of $H_{\mathrm{PL}}(X)$, we can construct such a map (cf. the proof of [GH, Theorem 2]). Thus we have the following version of Theorem 2.3.

2.5. THEOREM. Let $X$ be a positive dimensional compact Euclidean polyhedron. If $H(X)$ is an s-manifold and $\overline{H_{\mathrm{PL}}(X)}=H^{*}(X)$ then 
$\left(H^{*}(X), H_{\mathrm{PL}}(X)\right)$ is an $(s, \sigma)$-manifold pair and $\left(H^{*}(X), H_{\mathrm{LIP}}^{*}(X)\right)$ is an $(s, \Sigma)$-manifold pair.

We close the paper by posting the following

2.6. Conjecture. Let $X$ be a positive dimensional compact PL manifold in $\mathbf{R}^{m}$. Then $\left(H^{*}(X), H_{\mathrm{LIP}}^{*}(X), H_{\mathrm{PL}}(X)\right)$ is an $(s, \Sigma, \sigma)$-manifold triple. (Note that $H^{*}(X)=H(X)$ in case $\operatorname{dim} X \leq 3$ [Ra, Bi].)

\section{REFERENCES}

[An $\mathrm{A}_{1}$ R. D. Anderson, Hilbert space is homeomorphic to the countable infinite product of lines, Bull. Amer. Math. Soc., 72 (1966), 515-519.

[An $\mathrm{An}_{2}{ }_{-}$On sigma-compact subsets of infinite-dimensional spaces, unpublished manuscript.

[An $\mathrm{An}_{3}$, Spaces of homeomorphisms of finite graphs, unpublished manuscript.

[Bi] R. H. Bing, Locally tame sets are tame, Ann. of Math., (2) 59 (1954), 145-158.

[Br] E. M. Brown, The Hauptvermutung for 3-complexes, Trans. Amer. Math. Soc., 144 (1969), 173-196.

[Ch] T. A. Chapman, Dense sigma-compact subsets of infinite-dimensional manifolds, Trans. Amer. Math. Soc., 154 (1971), 399-426.

[Ga] D. B. Gauld, Local contractibility of spaces of homeomorphisms, Compositio Math., 32 (1976), 3-11.

[Ge $\left.{ }_{1}\right]$ R. Geoghegan, On spaces of homeomorphisms, embeddings, and functions-I, Topology, 11 (1972), 159-177.

[Ge $\left.{ }_{2}\right]$ On spaces of homeomorphisms, embeddings, and functions, II: The piecewise linear case, Proc. London Math. Soc., (3) 27 (1973), 463-483.

[GH] R. Geoghegan and W. E. Haver, On the space of piecewise linear homeomorphisms of a manifold, Proc. Amer. Math. Soc., 55 (1976), 145-151.

[Ha] W. E. Haver, Locally contractible spaces that are absolute neighborhood retracts, Proc. Amer. Math. Soc., 40 (1973), 280-284.

[Ja] W. Jakobsche, The space of homeomorphisms of a 2-dimensional polyhedron is an $l_{2}$-manifold, Bull. Acad. Polon. Sci. Sér. Sci. Math., 28 (1980), 71-75.

[KW] J. Keesling and D. C. Wilson, The group of PL-homeomorphisms of a compact PL-manifold is an $l_{2}^{f}$-manifold, Trans. Amer. Math. Soc., 193 (1973), 249256.

[LM] R. Luke and W. K. Mason, The space of homeomorphisms on a compact twomanifold is an absolute neighborhood retract, Trans. Amer. Math. Soc., 164 (1972), 275-285.

[LV] J. Luukkainen and J. Väisälä, Elements of Lipschitz topology, Ann. Acad. Sci. Fenn. Ser. A. I. Math., 3 (1977), 85-122.

[Mo] M. Morse, A special parameterization of curves, Bull. Amer. Math. Soc., 42 (1936), 915-922.

[Ra] T. Radó, Über den Begriff Reimannschen Fläche, Szeged Univ. Act., 2 (1925), 101-121.

[Sa $\left.\mathrm{S}_{1}\right]$ K. Sakai, On topologies of triangulated infinite-dimensional manifolds, J. Math. Soc. Japan, 39 (1987), 287-300. 
$\left[\mathrm{Sa}_{2}\right] \ldots, A Q$-manifold local-compactification of a metric combinatorial $\infty$ mani-

fold, Proc. Amer. Math. Soc., 100 (1987), 775-780.

[SW] K. Sakai and R. Y. Wong, On infinite-dimensional manifold triples, in preparation.

[SS] L. Siebenmann and D. Sullivan, On complexes that are Lipschitz manifolds, in: J. C. Cantrell, ed., Geometric Topology (Academic Press, New York, 1979), 503-525.

[Su] D. Sullivan, Hyperbolic Geometry and Homeomorphisms, Geometric Topology, Academic Press, New York, 1979, 543-555.

[To] H. Toruńczyk, Absolute retracts as factors of normed linear spaces, Fund. Math., 86 (1974), 53-67.

Received November 30, 1987 and in revised form June 8, 1988. This work was done while the first author was visiting the University of California at Santa Barbara, 1986.

UNIVERSITY OF TSUKUBA

TSUKUBA, IBARAKI

305 JAPAN

AND

UNIVERISTY OF CALIFORNIA

SANTA BARBARA, CA 93106

U.S.A. 
